2. Wada S, Noguchi T, Hashimoto T, Uchida Y, Kawahara K. Successful treatment of a patient with penetrating injury of the esophagus and brachiocephalic artery due to migration of Kirschner wires. Ann Thorac Cardiovasc Surg. 2005;11:313-5.

3. Venissac N, Alifano M, Dahan M, Mouroux J. Intrathoracic migration of Kirschner pins. Ann Thorac Surg. 2000;69:1953-5.
4. Nakayama M, Gika M, Fukuda H, Yamahata T, Aoki K, Shiba S, et al. Migration of a Kirschner wire from the clavicle into the intrathoracic trachea. Ann Thorac Surg. 2009;88:653-4.

5. Schwartz A, Thumerel M, Delcambre F, Jougon J. Transaortic migration of a Steinman wire from the shoulder. Eur J Cardiothorac Surg. 2011;40:517-9.

\title{
Amplatzer device migration through the sternum: A rare complication of percutaneous treatment for an aortic pseudoaneurysm solved by 2 length-adjustable bovine pericardium conduits
}

Ignacio Bibiloni Lage, MD, Stefano Benussi, MD, Alessandro Verzini, MD, and Ottavio Alfieri, MD, Milan, Italy

Pseudoaneurysm of the thoracic aorta after surgery is rare and life-threatening and involves hazardous surgery. ${ }^{1-4}$ Percutaneous strategies may reduce this risk but longterm results are limited. ${ }^{2,3}$ We report a replacement of an aortic graft using 2 bovine pericardial conduits after a failed percutaneous sealing of a perianastomotic thoracic aneurysm resulting from migration of an Amplatzer device (AGA Medical Corporation, Plymouth, Minn).

\section{CLINICAL SUMMARY}

A 43-year-old woman with Takayasu arteritis was admitted with new-onset chest pain 4 months after she had undergone percutaneous closure of a perianastomotic peudoaneurysm. During this period she had remained asymptomatic.

At the age of 30 years she had replacement of the ascending aorta for aortic aneurysm.

Four months earlier, during the study of a 7-year-old cutaneous fistula at the sternotomy wound, 3-dimensional computed tomography (CT) scan revealed a pseudoaneurysm at the distal graft anastomosis $(20 \times 15 \mathrm{~mm}$ with an 8-mm neck), unrelated to the fistula, which had eroded the sternum. Because the operative risk was considered high and cultures were negative without any signs of active infection, the pseudoaneurysm was sealed with a $10-\mathrm{mm}$

\footnotetext{
From the Division of Cardiac Surgery, San Raffaele University Hospital, Milan, Italy. Disclosures: Authors have nothing to disclose with regard to commercial support.

Received for publication Feb 3, 2012; revisions received April 16, 2012; accepted for publication May 15, 2012; available ahead of print June 14, 2012.

Address for reprints: Ignacio Bibiloni Lage, MD, Division of Cardiac Surgery,

San Raffaele University Hospital, Via Olgettina 60, 20132, Milan, Italy

(E-mail: igbibiloni@gmail.com).

J Thorac Cardiovasc Surg 2012;144:e27-8

$0022-5223 / \$ 36.00$

Copyright (C) 2012 by The American Association for Thoracic Surgery

http://dx.doi.org/10.1016/j.jtcvs.2012.05.033
}

Amplatzer muscular ventricular septal defect device. A proximal smaller pseudoaneurysm was left untreated (Figure 1, A).

When she was readmitted, still without signs of infection, CT showed the device in the pseudoaneurysm apex, like an arrowhead, perforating part of its wall (Figure 1, B). Echocardiography revealed mild aortic insufficiency. A surgical approach was therefore imperative.

The right axillary artery and the femoral vein were cannulated. A left ventricular vent was placed through the apex to prevent ventricular distention via submammary minithoracotomy. Cardiopulmonary bypass was established and bladder temperature was lowered to $20^{\circ} \mathrm{C}$. Corticosteroids and thiopental were used for neuroprotection.

During cooling, according to the aortic graft diameter measured by CT, we made 2 bovine pericardium conduits using as a mold a Hegar dilator, each with a double suture of 5-0 polypropylene tied at only one end in order to adjust their lengths afterward (Figure 2, A)

Under circulatory arrest, resternotomy was undertaken resulting in the rupture of both pseudoaneurysms (Figure 2, $B$ and $C$ ). The previous Dacron graft was removed and antegrade cerebral perfusion was administered through the axillary and the left carotid arteries at $800 \mathrm{~mL} / \mathrm{min}$ and $400 \mathrm{~mL} / \mathrm{min}$, respectively, while the innominate and the left subclavian arteries were occluded. The distal aorta was anastomosed to one of the readymade conduits and CBP was resumed after the distal graft had been clamped. The second conduit was sutured to the sinotubular junction and the 2 conduits were anastomosed to each other with 4-0 polypropylene. Cardiopulmonary bypass and circulatory arrest times were 203 minutes and 46 minutes, respectively.

Staphylococcus epidermidis grew on the culture of the graft biopsy. The patient was discharged on postoperative 

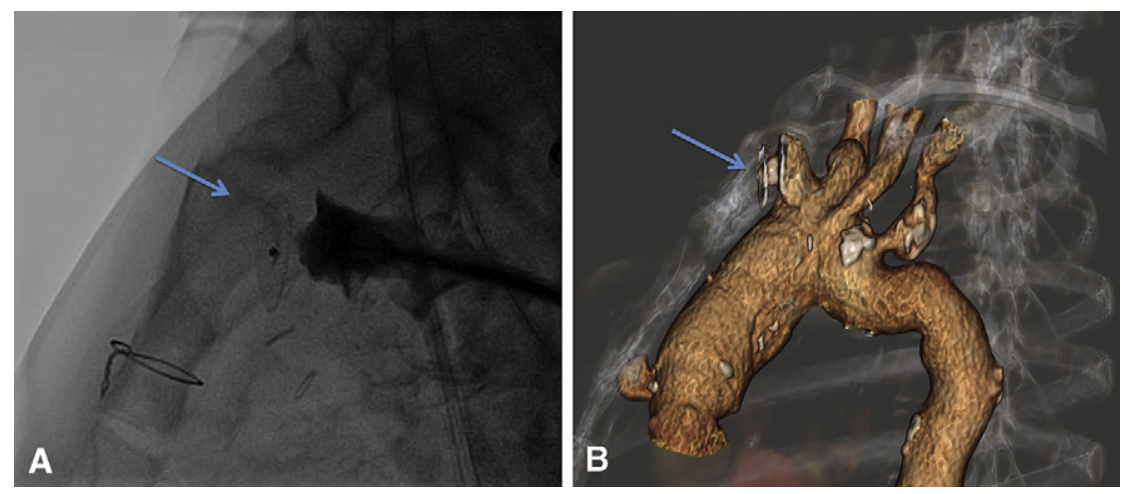

FIGURE 1. A, Angiogram. Successful sealing of the pseudoaneurysm by the Amplatzer device (arrow). B, Computed tomographic scan. The pseudoneurysm erodes the sternum with the device (arrow) in its apex.
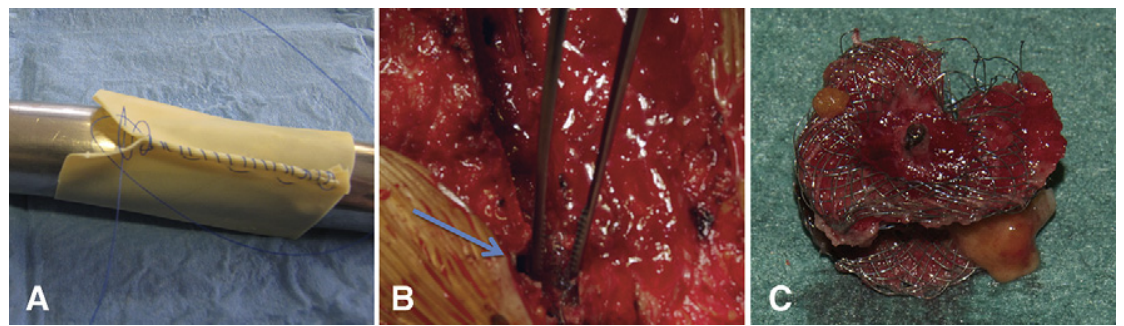

FIGURE 2. A, Construction of pericardial grafts. B, The device (arrow) is shown after resternotomy. C, Amplatzer device.

day 10 receiving antibiotics. CT revealed an optimum result.

\section{COMMENT}

Pseudoaneurysm of the thoracic aorta occurs in less than $0.5 \%$ of cardiac surgery cases, and infection, usually owing to Staphylococcus species, is an important predisposing factor. ${ }^{1,4}$ Pseudoaneurysm has also been associated with Takayasu arteritis. ${ }^{5}$

Resternotomy may be associated with high morbidity and mortality owing to pseudoaneurysm rupture. ${ }^{1-5}$ Although long-term follow-ups need to be reported, Amplatzer devices have been used successfully to occlude aortic pseudoaneurysms. $^{2,3,5}$ These reasons, in addition to the absence of signs of infection and her wound healing problems, led us to use this approach. We are confident that Amplatzer migration was not due to technical reasons as we measured the pseudoaneurysm by CT and echocardiography, and the oversized device was deployed without misalignment problems or residual shunt under echocardiographic and fluoroscopic guidance. We hypothesize than both infection and arteritis could have played a role in this case.

Despite the absence of sepsis and repeated negative cultures, the possibility that the fistula could act as an infectious focus, her immunosuppressive therapy for Takayasu arteritis, and the prevalence of infectious etiology ${ }^{1,4,5}$ made us act as though it were an infected pseudoaneurysm. Consequently, bovine pericardium was preferred. However, we cannot discard the possibility that the intraoperative culture was a contaminant.

\section{CONCLUSIONS}

From our limited experience, we think that surgery should be the first choice for perianastomotic thoracic pseudoaneurysms except in patients with multiple comorbidities until predisposing factors for percutaneous approach failure are clarified.

Length-adjustable grafts could be useful tools for this surgery, and bovine pericardium could be a good option when an infectious etiology is suspected.

We thank Dr Enrico Rinaldi and Dr Federico Anzil for their assistance.

\section{References}

1. Sullivan KL, Steiner RM, Smullens SM, Griska L, Meister SG. Pseudoaneurysm of the ascending aorta following cardiac surgery. Chest. 1988;93: 138-43.

2. Noble S, Ibrahim R. Embolization of an Amplatzer mVSD occluder device used for percutaneous closure of an ascending aortic pseudoaneurysm: case report and literature review. Catheter Cardiovasc Interv. 2012;79:334-8.

3. Hussain J, Strumpf R, Wheatley G, Diethrich E. Percutaneous closure of aortic pseudoaneurysm by Amplatzer occluder device - case series of six patients. Catheter Cardiovasc Interv. 2009;73:521-9.

4. Atik FA, Navia JL, Svensson LG, Vega PR, Feng J, Brizzio ME, et al. Surgical treatment of pseudoaneurysm of the thoracic aorta. J Thorac Cardiovasc Surg. 2006;132:379-85.

5. Malvindi PG, van Putte BP, Heijmen RH, Schepens MA, Morshuis WJ. Reoperations for aortic false aneurysms after cardiac surgery. Ann Thorac Surg. 2010;90: $1437-43$. 